\title{
SOCIOLOGIE SÉMANTICO-LOGIQUE DES RUINES: POUR UNE HERMÉNEUTIQUE HYBRIDE DE LA RUINE DU WEB 2.0 AU WEB 3.0
}

\author{
Pedro Andrade
}

De Boeck Supérieur | Sociétés

$2013 / 2-n^{\circ} 120$
pages 105 à 119

ISSN 0765-3697

Article disponible en ligne à l'adresse:

http://www.cairn.info/revue-societes-2013-2-page-105.htm

Pour citer cet article :

Andrade Pedro, « Sociologie sémantico-logique des ruines: pour une herméneutique hybride de la ruine du Web 2.0 au Web 3.0 ",

Sociétés, $2013 / 2$ n¹20, p. 105-119. DOI : 10.3917/soc.120.0105

Distribution électronique Cairn.info pour De Boeck Supérieur.

(c) De Boeck Supérieur. Tous droits réservés pour tous pays.

La reproduction ou représentation de cet article, notamment par photocopie, n'est autorisée que dans les limites des conditions générales d'utilisation du site ou, le cas échéant, des conditions générales de la licence souscrite par votre établissement. Toute autre reproduction ou représentation, en tout ou partie, sous quelque forme et de quelque manière que ce soit, est interdite sauf accord préalable et écrit de l'éditeur, en dehors des cas prévus par la législation en vigueur en France. II est précisé que son stockage dans une base de données est également interdit. 


\title{
SOCIOLOGIE SÉMANTICO-LOGIQUE DES RUINES : POUR UNE HERMÉNEUTIQUE HYBRIDE DE LA RUINE DU WEB 2.0 AU WEB 3.0
}

Pedro ANDRADE *

\begin{abstract}
Résumé : Le terme ruine renvoie, à la fois, à un processus social complexe et à un concept polysémique. Les ruines constituent également un phénomène qui a une nature relationnelle. Par conséquent, une étude sociologique possible se constitue à partir des deux piliers suivants : (a) des concepts interprétés en utilisant une posture sémantique à l'égard du social et du sociologique; (b) des relations sociales de nature logique et hybride. Ce texte développe ce type de réflexion en utilisant les outils théoriques et méthodologiques construits au sein d'un paradigme sociologique nommé Sociologie sémantico-logique et à l'intérieur de l'une de ses tactiques, l'Hybridologie sociale, qui utilise en particulier un style d'interprétation désigné Herméneutique hybride. Cette méthodologie est appliquée à l'analyse de contenu et de discours de la page "Ruins " de Wikipédia, en essayant d'y extraire les sens polymorphes qui traduisent l'imaginaire subjacent à la forme sociale "ruine».
\end{abstract}

Mots clés : ruine, sociologie sémantico-logique, hybridologie sociale, herméneutique hybride

\footnotetext{
Abstract: The term ruin refers to both a complex social process and a polysemic concept. Ruins may also be considered a phenomenon that has a relational nature. Consequently, a sociological study is to be based on two pillars: (a) concepts that are interpreted using a semantical posture towards the social and the sociological, and (b) social relationships presenting both a logic and hybrid nature. This text develops such reflection bases, using theoretical and methodological tools built within a sociological paradigm called SemanticLogic Sociology, and inside of one of its tactics, Social Hybridology, that uses a particular style of interpretation, named Hybrid Hermeneutics. Such methodology is applied to the

* Chercheur au Centro de Estudos de Comunicação e Sociedade, Université de Minho, Portugal.
} 
content and discourse analysis of the Wikipedia page "Ruins », trying to extract the polymorphic meanings that reflect the imaginary subjacent to social form ruin.

Keywords: ruin, semantic-logic sociology, social hybridology, hybrid hermeneutics

\section{Introduction : la Sociologie sémantico-logique, un paradigme pour l'analyse des ruines}

Le terme ruine renvoie, à la fois, à un processus social complexe et à un concept polysémique : il suffit de consulter ce mot à l'intérieur d'un Dictionnaire de langue ou dans un Thesaurus ou autre lexique spécialisé de concepts, pour s'apercevoir de la richesse de sens qu'il possède. Les ruines constituent également un phénomène qui a une nature relationnelle. Elles s'inscrivent au sein des relations sociales d'une civilisation, d'une culture ou d'une communauté. Ces relations sociales se manifestent, en partie, en tant que relations logiques, mais également comme des rapports d'hybridation ou de fusion parmi des imaginaires et de plusieurs formes sociales différentes, à l'intérieur de notre monde interculturel. Par conséquent, une étude sociologique sur les ruines peut se constituer à partir des deux piliers suivants : (a) des concepts définis en utilisant une posture interprétative sémantique à l'égard du social et du sociologique ; (b) l'analyse de relations sociales de nature logique et hybride. Ce texte développe cette réflexion en utilisant les outils théoriques et méthodologiques construits dans le cadre d'un paradigme sociologique nommé Sociologie sémantico-logique. Un tel style d'écriture sociologique se fonde sur les présupposés suivants.

\section{Théorie, concepts, méthodologie}

Nos pratiques et imaginaires sociaux se trouvent ancrés, en grande partie, dans le cyberespace et, en particulier, au sein du Web 2.0. Ce dernier à considérer comme une sorte de seconde ère de l'Internet, qui a connu un développement remarquable depuis 2001, et qui présente une forme sociale singulière, caractérisée par deux attributs centraux :

(a) Le citoyen est censé être plus actif que durant la décennie précédente. En d'autres mots, à la dernière décennie du $\mathrm{XX}^{e}$ siècle, l'utilisateur du cyberespace se concentrait dans la simple lecture des informations, même s'il pouvait pratiquer une écriture basique, comme un texte dans un e-mail, ou parfois des textes plus élaborés dans une web page, mais néanmoins en sens unique. Au contraire, à la décennie suivante, l'utilisateur peut écrire plus de messages à partir d'instruments et dispositifs discursifs divers, comme un blog ou un wiki. C'est pourquoi le Web 2.0 est également appelé «Web social de lecture/ écriture ».

(b) Les réseaux sociaux se globalisent. Il ne s'agit plus du réseau spécialisé d'une université ou d'une entreprise, mais le citoyen commun peut participer et partager ses lectures ou ses écrits en ligne.

Cependant, de nos jours, nous pouvons apercevoir des signes d'une autre façon de naviguer ou de chercher/partager de l'information sur Internet. Ce processus, 
appelé « Web 3.0 », constitue une très récente forme sociale, comportant ce trait central : un nouveau style de production et de réception pas seulement d'informations, mais surtout de connaissances et de sens. Souvent, le Web 3.0 est appelé "Web sémantique », en ce que son objectif principal est de construire des sites et des réseaux où leur propre paradigme ou modèle de signification et de connaissance est manifeste et/ou décrit parfois en détail. En d'autres termes, dans ces " sites sémantiques », l'information est organisée autour d'ontologies, qui peuvent être définies comme des modes d'organisation des significations, consistant, entre autres instruments, en des ensembles de concepts et de relations sémantiques et logiques mis en place entre les éléments ou pôles d'information. Dans le Web 2.0, certains de ces concepts et relations sont déjà construits manuellement, comme les tags (mots, termes ou concepts descriptifs) que l'utilisateur peut associer, par exemple, à un texte sur un blog. Ces tags constituent du metadata, c'est-à-dire une information synthétisée sur l'information originale. Dans le Web 3.0 sémantique, l'utilisation des métadonnées est plus répandue que dans le Web 2.0. Actuellement, on peut trouver plusieurs exemples de sites sémantiques dans le cyberespace et dans le cybertemps (c'est-à-dire les temporalités sous-jacentes au cyberespace) comme Freebase, Semantic Media Wiki, etc.

Létude sociologique systématique de ces formes sociales centrales a déjà été entreprise, mais, surtout dans le cas du Web 3.0, les essais sont encore rares. Dans une réflexion sur l'articulation entre le Web 2.0 et le Web $3.0^{1}$, se présente une théorisation de ce processus et de son application au moyen d'analyses de contenu et de discours particulières sur divers sites au sein du Web 2.0, nommées analyses sémantico-logiques. Comme il y est démontré, la recherche dans les sciences sociales est en mesure de développer des méthodologies sémantico-logiques. Ces méthodes peuvent se comprendre comme un ensemble de procédures particulières de lecture et d'écriture sociologiques à l'égard de textes, images, vidéos, animations, contenus et interfaces inter-media et performatifs, travaillant au Web 2.0 et 3.0. Concrètement, ces procédures considèrent soit les sources classiques, soit les sources digitales, en hybridant souvent ces deux types de sources. Dans le cas des sciences sociales et humaines, une telle lecture/écriture sociologique hybride se passe à travers l'interprétation du sens sémantique et logique profond des formes sociales, construisant ainsi des réseaux sémantico-logiques qui traduisent les pratiques de l'imaginaire sociétal.

Bref, la Sociologie sémantico-logique est définie comme une stratégie et un style d'enseignement et de recherche en sociologie (et, plus généralement, dans les sciences sociales) ayant une nature à la fois différentielle et fusionnelle, un trait qui caractérise aussi bien le Web 2.0 que le Web 3.0. En effet, en ce qui concerne le Web 2.0, sa caractéristique repose sur le partage collectif d'informations diverses parmi les acteurs sociaux. À son tour, le Web 3.0 mobilise la capacité de production

1. P. Andrade, Sociologia Semântico-Lógica da Web 2.0/3.0 na sociedade da investigação: significados e discursos quotidianos em blogues, wikis, mundos/museus virtuais e redes sociais semântico-lógicas, Caleidoscópio, Lisbonne, 2011, pp. 169-205. 
de sens/connaissances communes et d'établissement de relations logiques partagées et souvent « hybridisées », parmi des acteurs sociaux singuliers. De ce fait, les agents ne se trouvent pas seulement inscrits au sein des réseaux sociaux, mais aussi écrits et réécrits par et pour tous ses membres.

Cependant, ces deux styles de participation se distinguent par des spécificités. Si le Web 2.0 travaille comme un Web social, le Web 3.0 fonctionne principalement comme un Web sémantique. En d'autres mots, le Web 3.0 investit surtout sur la production de sens social interprétatif ou explicatif, et pas seulement sur un sens social descriptif ou narratif, comme pour le Web 2.0. Le Web 3.0 se présente donc en tant que Web sociologique ou Web socio-logique, en ce qui concerne le partage d'informations et de connaissances, qu'il maintient mais dans un style distinct du Web 2.0. Mais comment pouvons-nous construire, d'une manière concrète, des outils théoriques et méthodologiques qui nous permettent d'appliquer la Sociologie sémantico-logique, par exemple aux réseaux sociaux du Web 2.0 et du Web 3.0 ?

\section{L'Hybridologie sociale}

À partir de la déconstruction des savoirs actuels, on assiste aujourd'hui à la reconstruction de la connaissance en des figures originales. Lune des figures de ce que nous appelons lecture/écriture sociologique expérimentale, en particulier en ce qui concerne la Sociologie sémantico-logique, est l'Hybridologie.

Avant tout, l'Hybridologie est associé à la notion d'hybride. Elle consiste dans un mode heuristique de lecture/écriture, simultanément réflexive et intuitive, qui met l'accent sur des entités et des identités hybrides qui se multiplient à l'époque contemporaine. Lorsque l'on étudie une société donnée, par exemple les flux d'hybridation à l'intérieur des réseaux sociaux, l'Hybridologie prend le nom d'Hybridologie sociale. La condition de l'hybride est souvent connotée avec les idées de mélange, hétérogénéité, métissage, monstruosité, etc. Toutefois, au sein du cyberespace, et au-delà de la blogosphère, aujourd'hui une hybridosphère émerge. L'un des traits de cette nouvelle forme sociale est la fusion de la nature du cyberespace avec les racines du cybertemps. 
Table 1: distribution du mot-clé 'Ruine' dans l'ensemble de la page 'Ruins' (Wikipedia)

\begin{tabular}{l}
$\begin{array}{l}\mathrm{N}^{\circ} \text { de } \\
\text { phrase }\end{array}$ Texte de la phrase \\
\hline
\end{tabular}

1 Introduction: RUINEs.

2 Image: RUINEs du Forum romain à Rome.

4 Les RUINEs sont les vestiges de l'architecture d'origine humaine:

17 Il y a des RUINEs célèbres partout dans le monde,

19 Les RUINEs sont d'une grande importance pour les historiens, les archéologues et les anthropologues:

25 Image: Tremblement de terre à San Francisco,1906: RUINEs dans les environs de Post et Grant Avenue.

29 Bien que moins central dans les conflits modernes, de vastes zones des villes du 20e siècle, telles que Varsovie, Dresde, Coventry, Londres et Berlin, ont été laissés en RUINEs après la Seconde Guerre mondiale.

La ville antique de Pompéi a intégralement disparue lors d'une éruption volcanique dans le 1er siècle, et, après la découverte de ses RUINEs, ont été conservées comme site du patrimoine mondial.

La ville de Lisbonne a été totalement détruite en 1755 par un violent séisme et un tsunami, et le tremblement de terre de 1906 à San Francisco a quitté la ville en RUINE presque complète.

Image 2: Winston Churchill visitant les RUINEs de la cathédrale de Coventry après le Blitz à Coventry au moment de la Seconde Guerre mondiale.

De nombreuses abbayes et monastères tombèrent en RUINEs lorsque leurs biens, y compris les toits de plomb, ont été dépouillés.

Après la Seconde Guerre mondiale, un certain nombre de bâtiments historiques européennes est tombé en RUINE à cause de politiques fiscales,

L'Irlande post-coloniale a encouragé la RUINE de grandes demeures georgiennes, symboles de l'impérialisme britannique. [7]

54 Image: RUINEs sur-mesure: la "RUINE romaine" dans le parc de Schönbrunn, c 1800.

60 La contemplation des RUINEs de la "ceinture de rouille" post-industrielle est à ses balbutiements. [8]

63 Image: Les RUINEs du Parthénon à Athènes, en Grèce.

Au Moyen Age, les RUINEs romaines étaient des obstacles gênants à la vie moderne, des carrières de blocs préformés pour des projets de construction, des marbres à être brûlés pour la chaux agricole, des sujets de commentaires satisfaisants sur le triomphe du christianisme, et le sens général du déclin du monde, dans ce qui était supposé être son dernier âge, avant la Seconde Venue.

Avec la Renaissance, les RUINEs reçoivent de nouveaux rôles à l'intérieur d' une élite culturelle, comme des exemples d'une architecture délibérément relancé et purifié all' antica, et pour une nouvelle appréciation esthétique de leur beauté innée en tant qu' objets de décadence vénérable. [9]

conduit certains artistes et designers de cette période à concevoir des monuments classiques modernes comme s'ils seraient un jour apparus en tant que RUINEs.

La valeur RUINE (en allemand: RUINEnwert) est le concept qu'un bâtiment soit conçu de telle sorte que, s'il finissait par s'effondrer, il laisserait derrière lui des RUINEs esthétiquement agréables, qui allaient durer beaucoup plus longtemps sans aucun entretien du tout.

Joseph Michael Gandy paint pour Sir John Soane en 1832 une aquarelle atmosphérique de la vaste rotonde de la Banque d' Angleterre comme une RUINE énorme et pittoresque, qui est une icône du romantisme. [10]

RUINEnwert a été popularisé au 20e siècle par Albert Speer, pendant la planification des Jeux olympiques de l'été de 1936, et publié en tant que Die RUINEnwerttheorie ("La théorie de la valeur RUINE").

Les RUINEs restent un sujet populaire pour la peinture et la photographie créatives [11] et sont souvent romancées dans les films et la littérature, en fournissant des décors scéniques ou utilisés comme des métaphores pour d'autres formes de déclin ou de décadence. tableaux; en 1989, les RUINEs du château de Dunnottar en Ecosse ont été utilisées pour le tournage de Hamlet. 
LHybridologie sociale est aussi étroitement liée à l'idée de littératie (literacy), parmi d'autres connexions conceptuelles. La littératie ne peut être limitée à l'idée d'alphabétisation tout court, mais doit est considérée dans un sens élargi, comme un ensemble de stratégies de lecture et d'écriture subjacentes à un mode de savoir donné. En plus des régimes de lecture et d'écriture propres à une langue nationale, on assiste aujourd'hui à la prolifération de littératies diverses, inhérente aux multiples activités et langages opérant dans la contemporanéité, dont la langue nationale n'est qu'un cas particulier. Ainsi, il est possible de parler de littératies scientifique, technologique ou artistique.

Lun de ces modes de savoir est le sens commun ou connaissance ordinaire du quotidien ${ }^{2}$. Chaque acteur social intériorise le savoir spécifique d'un rôle social ou d'une profession, où des argots spécialisés peuvent se structurer. Néanmoins, quand des acteurs communiquent avec d'autres acteurs hors le contexte d'une spécialité langagière donnée, ils le font en utilisant un langage commun, celui de la vie quotidienne collective. Cette littératie ordinaire de l'espace public de la ville présente des formes partagées de lecture/écriture (ex. la lecture d'un horaire d'autobus) et sert souvent de liaison ou de dénominateur commun à toutes les autres littératies, en les hybridant parfois. En particulier, le cyberespace permet de nouvelles formes d'habiter et de construction de l'imaginaire social ${ }^{3}$. Au sein des réseaux sociaux digitaux, cette littératie collective du citoyen ordinaire acquiert une importance centrale. Par exemple, dans Wikipédia, tout le monde peut participer à la construction de concepts communs, même si ces contenus sont évalués par des commissions de lecture qui veulent assurer la qualité de l'information.

Enfin, quelles stratégies pratiques peut-on utiliser afin d'appliquer la Sociologie sémantico-logique et en particulier l'Hybridologie sociale au sujet d'étude «ruines"?

\section{l'herméneutique hybride des ruines}

Lune des tactiques analytiques et interprétatives qui opérationnalisent la stratégie théorico-méthodologique désignée auparavant comme Hybridologie sociale, est l'herméneutique hybride. Celle-ci sert, entre autres objectifs, à effectuer l'analyse de contenu et/ou de discours sur les processus sociaux hybrides. Pour ce faire, cet article interprétera une page de l'encyclopédie digitale Wikipédia ayant le titre Ruins, dans sa version en langue anglaise ${ }^{4}$. Vu l'espace restreint de cet article, nous ne sélectionnerons ici que deux sections de la page, qui correspondent à deux champs sémantiques à l'intérieur de l'argumentation du texte : la première section

2. M. Maffesoli, La passion de l'ordinaire : Miettes sociologiques, CNRS Éditions, Paris, 2011.

3. F. La Rocca, " Habitar a Web: paisagens e nuvens da cultura digital », Revista de Comunicação e Linguagens: Genealogias da Web 2.0, 42, 2001, p. 53.

4. "Ruins" (2012), in Wikipédia, consulté en ligne le 20 octobre 2012, permalien : http:// en.wikipedia.org/wiki/Ruins. 
introduit le concept de ruine, et a été nommée "Ruines ", ainsi que le champ sémantique respectif ${ }^{5}$. Le deuxième, qui se réfère aux aspects esthétiques de la ruine, sera reprise plus tard.

Tout d'abord, si l'on cherche les occurrences du mot « ruine » dans le texte de la page de Wikipédia, on obtient les extraits suivants (traduits en français pour la commodité de la lecture), où l'on constate les usages sémantiquement pluriels du mot (cf. tableau 1).

Néanmoins, une analyse qualitative ne portant que sur les notions isolées de leurs relations, s'avère souvent incomplète. Différemment, nous considérerons les réseaux sémantico-logiques qui organisent les principaux concepts et leurs relations sur la page de l'internet sélectionnée (cf. figure 1).

Figure 1. Réseau sémantico-logique du champ sémantique 1 nommé « Ruines » (2D)

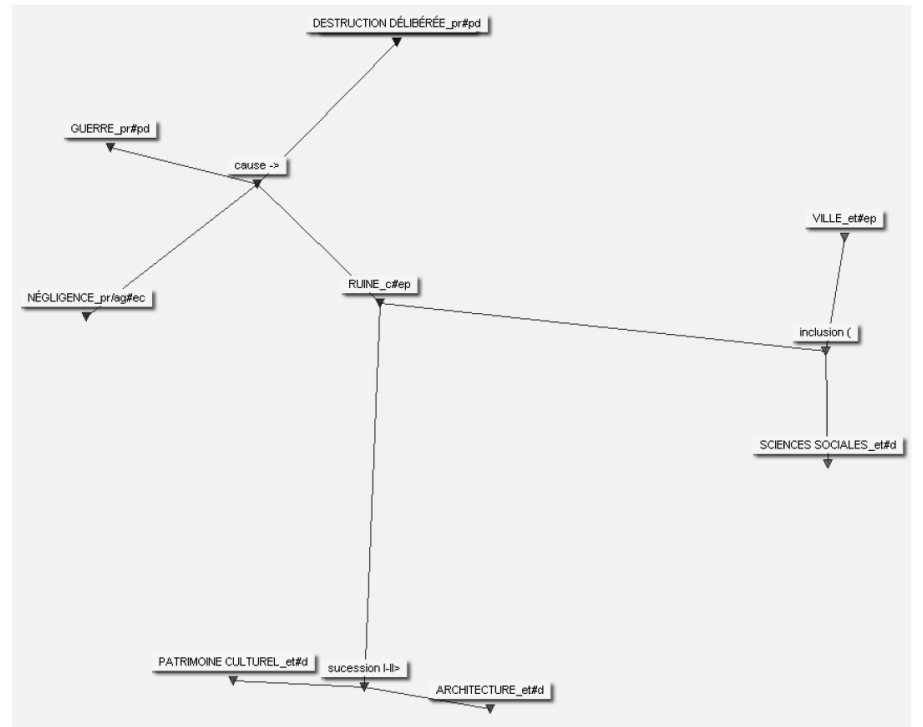

5. Voici son texte original dans la page : "Ruins are the remains of human-made architecture: structures that were once complete, as time went by, have fallen into a state of partial or complete disrepair, due to lack of maintenance or deliberate acts of destruction. Natural disaster, war and depopulation are the most common root causes, with many structures becoming progressively derelict over time due to long-term weathering and scavenging. There are famous ruins all over the world, from ancient sites in China, the Indus valley and Judea to Zimbabwe in Africa, ancient Greek, Egyptian and Roman sites in the Mediterranean basin, and Incan and Mayan sites in the Americas. Ruins are of great importance to historians, archaeologists and anthropologists, whether they were once individual fortifications, places of worship, houses and utility buildings, or entire villages, towns and cities. Many ruins have become UNESCO World Heritage Sites in recent years, to identify and preserve them as areas of outstanding value to humanity." 
En d'autres mots, on se penchera surtout sur le sens profond des concepts, mais aussi sur les types de relations logiques qui les articulent. De même, nous utiliserons une approche parfois quantitative, hybridisée avec la posture qualitative précédente.

En ce qui concerne les idées centrales présentes dans le texte, elles peuvent être traduites, dans le travail herméneutique, par des concepts hybrides. Ceux-ci sont des concepts qui articulent et fusionnent plusieurs dimensions du social, à l'intérieur du definiendum même.

Figure 2. Réseau sémantico-logique du champ sémantique 1 « Ruines 》 (3D)

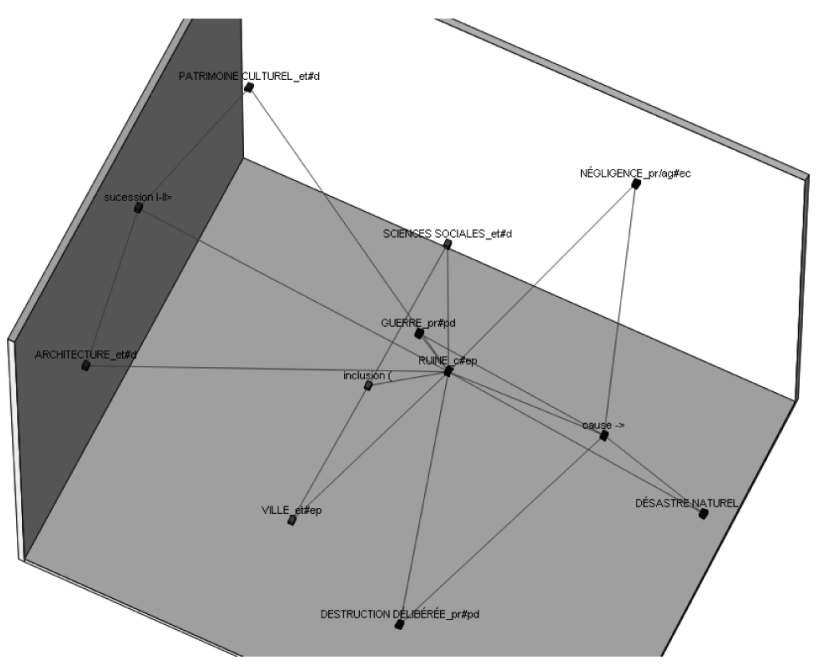

Le definiendum est le nom du concept qu'on a l'intention de définir. Et le definiens est la deuxième partie d'une définition, constituée par des mots/termes plus simples ou plus connus par le sens commun, ou encore définis précédemment, qui éclaircissent le concept plus complexe qu'on veut définir. Dans le cas des concepts hybrides, les notions qui aident à l'interprétation du sens du concept à définir, se situent au sein même du nom de ce concept.

Par exemple, le concept « ruine » (definiendum), dans une définition classique, peut être circonscrit, entre autres connotations, en utilisant un definiens qui inclut les mots familiers suivants : " objet ou édifice semi-détruit par l'œuvre du temps, de la guerre ou de l'incurie humaine ». Par contre, si on interprète le concept " ruine » à partir d'une définition issue de l'herméneutique hybride (c'est-à-dire en 
tant que concept hybride), nous obtiendrons ceci : l'idée ruine peut être comprise comme l'hybridation des dimensions sociales " espace » (le lieu, l'espace architectural), et le "contexte social » où elle se situe, qui articule cet espace local avec le temps social (institutionnel, etc.) et avec le temps historique. La notation respective (figure 1) est : 'ruine_c\#ep'. Le premier élément, « ruine » signifie l'idée que l'on veut définir. Le deuxième élément, 'c\#ep', séparé du premier par un trait ', ', est une codification qui lie, d'une part, le code associé au contexte social, 'c', à, d'autre part, le code de l'espace, 'ep'. Le tout constitue le concept hybride qui, dans ce cas, articulent deux dimensions.

Figure 3. Dendrogramme du champ sémantique I 'Ruins'

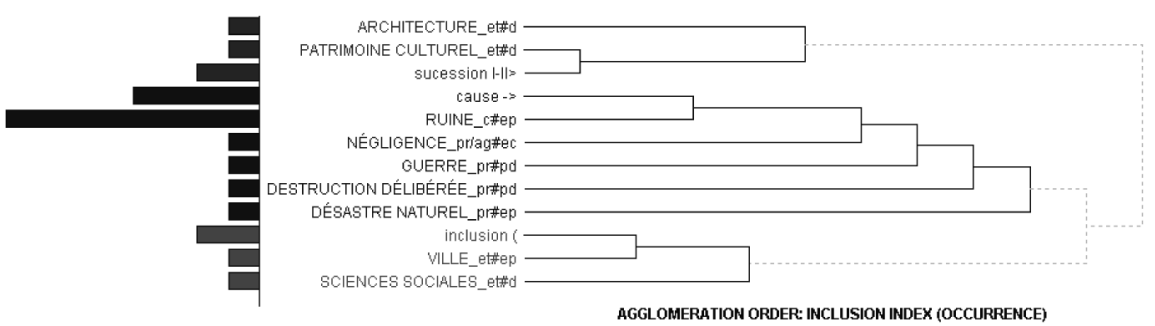

Pour ce qui est des relations logiques et hybrides, nous avons élaboré un alphabet de relations logiques universelles ${ }^{6}$. Cet alphabet réunit, dans un tout cohérent et codifié, des relations issues de la logique formelle (Aristote, Descartes), de la logique dialectique (Héraclite, Hegel, Marx), de la logique booléenne utilisée par les ordinateurs, de la logique fuzzy de l'Intelligence artificielle, et même de la logique analogique, qu'on peut trouver dans certains systèmes de pensée non rationnelle, irrationnelle ou antirationnelle. Notons que ces relations logiques peuvent se trouver hybridisées de plusieurs façons. Lune de ces manières est la fusion de la notion "relation logique » avec la notion même de ce qu'on entend par " concept ", dans la forme de la figure hybride de la connaissance nommée "relation-concept ${ }^{7}$. Nous donnerons ici quelques exemples de ces concepts et relations hybrides.

Pour appliquer l'herméneutique hybride à travers ces concepts et ces relations épistémologiquement revisitées, la première étape consiste à sélectionner des champs socio-sémantiques spécifiques ou à entreprendre l'interprétation. Dans cet article, ils coïncident grosso modo avec ses principales sections. Par exemple, considérons le champ sémantique 1 , associé à la section 1 , intitulée "Ruines »

6. P. Andrade, "O alfabeto das relações universais ", Revista de Comunicação e Linguagens, 38, 2007, pp. 148-153.

7. P. Andrade, "Some synthetic ideas to work with qualitative analysis ", Atalaia/Intermundos, 10/11, 2002, p. 160. 
(présentée auparavant à la note 5). Une exégèse possible du sens de ce champ sémantique est celle qu'on observe à la figure 2. Ce réseau social sémanticologique présente le concept hybride "ruine » à son centre. En fait, c'est le concept qui établit le plus de relations avec d'autres. Et il le fait à partir de trois sous-champs socio-sémantiques, chacun identifié par une couleur et ayant, comme concept médiateur, une relation-concept.

Par exemple, prenons le sous-champ sémantique associé à la relation-concept "succession I-II > ». Les éléments de ce sous-champ (concepts et relations) sont marqués en rouge dans l'image. La relation codée «I-II > » exprime la succession temporelle de deux phénomènes : elle articule le concept hybride 'ARCHITECTURE', codé 'ep\#d' (espace discursif), et 'RUINE', à travers un processus temporel. La phrase correspondante dans le texte du champ sémantique (note 5) est : "Ruins are the remains of human-made architecture » (Les ruines sont les vestiges de l'architecture d'origine humaine). Dans le même sous-champ sémantique, l'expression synthétique et canonique RUINE_c\#ep' succession I-II> PATRIMOINE CULTUREL_et\#d, signifie, dans le texte original : "Many ruins have become UNESCO World Heritage Sites in recent years » (De nombreux vestiges sont devenus des sites du patrimoine mondial de l'UNESCO ces dernières années). Le code 'et\#d' veut dire 'structure discursive'.

La dimension temporelle esquissée ci-dessus est une constante quand on parle des ruines. Toutefois, les ruines ne se réfèrent pas toujours au passé. Les ruines atomiques de Tchernobyl témoignent du péril constant, dans le monde actuel, de la possibilité pour ce monde présent de devenir du passé pour tout le futur. Santiago Camacho ${ }^{8}$ décrit la ville fantôme de Pripyat, édifiée pour les travailleurs de la centrale, devenue le meilleur monument qui alerte pour ce cauchemar.

Figure 4. Réseau sémantico-logique du champ sémantique 2 « Esthétique » (2D)

8. S. Camacl

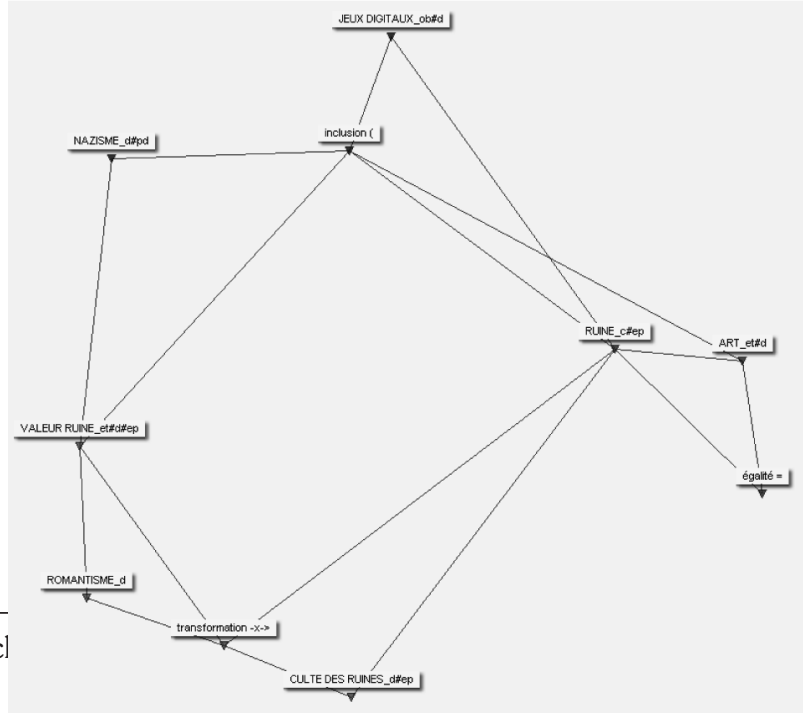

$? 011$.

Sociétés $n^{\circ} 120-2013 / 2$ 
Observons maintenant le cluster du sous-champ sémantique connecté à la relation sociale 'cause $\rightarrow$ ', indiqué en bleu. Son sens est résumé par la phrase synthétique suivante : la destruction délibérée, ou la guerre, ou encore la négligence, causent la ruine. Le code 'pr\#pd', lié à la 'guerre', a le sens de 'pratique de pouvoir'. À son tour, le code associé à 'négligence' (pr/ag\#ec) se traduit par une pratique d'un agent dans la sphère économique. Notons au passage que, tandis que le rapport entre deux dimensions se code '\#', le rapport entre deux sous-dimensions est représenté par ' $\%$ '. En fait, une pratique et un agent s'incluent dans le niveau microsociologique (une sous-dimension) à l'intérieur de la dimension " niveaux sociaux » (celle-ci sémantiquement plus générale), à laquelle appartiennent aussi le niveau macrosociologique et le niveau médiateur du social.

Nous trouvons ici l'un des sens profonds émis par les ruines : en général, elles deviennent un motif puissant d'attraction pour les hommes. En particulier, elles ont une double nature : le côté de l'ombre inhérente à l'existence humaine, et un outil critique et méthodologique pour interpréter cette vie humaine souvent en crise ${ }^{9}$.

Figure 5. Intensité sémantique des concepts et relations (2D)

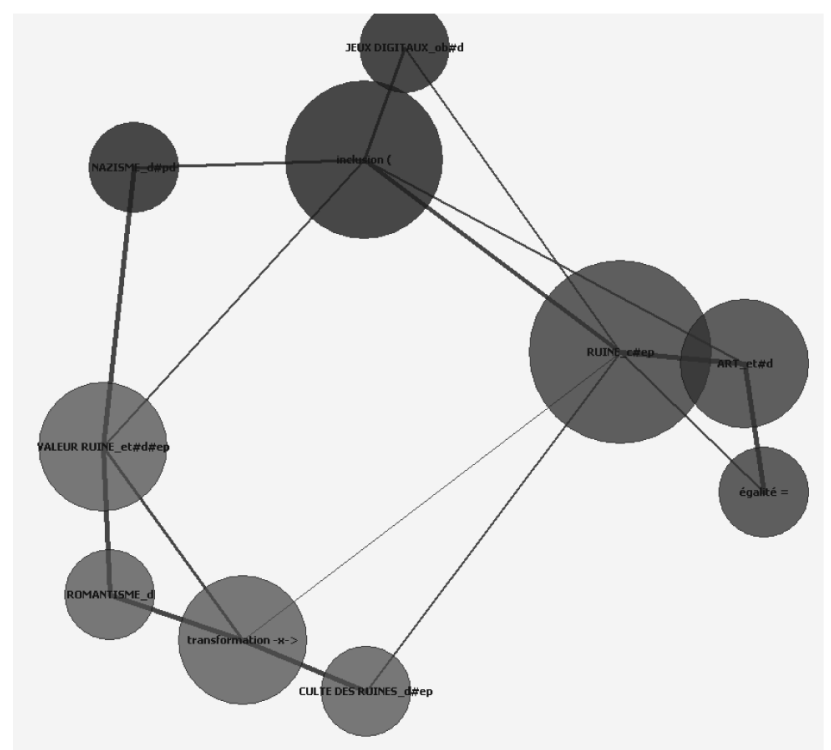

Le troisième sous-champ sémantique est celui lié à la relation-concept « inclusion ", et noté en vert. L'expression linguistique de ce rapport logique d'inclusion est la suivante : les villes incluent, à leur intérieur, maintes ruines, et les sciences

9. S. Lacroix, Ce qui nous disent les ruines : fonctions critiques des ruines, L'Harmattan, Paris, 2007. 
sociales s'intéressent aux ruines comme l'un de leurs objets d'études. Par exemple, la ville de Paris a produit des types sociaux dynamiques qui ont forgé un imaginaire social unique ${ }^{10}$. Mais la mort des villes, témoignée par les restes de la ville industrielle, servira aussi pour alimenter le culte des ruines ${ }^{11}$.

La figure 2 montre également les rapports du réseau à l'intérieur du champ sémantique "Ruines », mais cette fois représentée à travers un espace 3D.

Une autre perspective, manifestant une nature hybride entre le quantitatif et le qualitatif, est visible dans la figure 4. Ce dendrogramme explicite, à la droite, les relations qualitatives d'agglomération entre les concepts et les relations concernés. Et, en même temps, dans le graphique à gauche, le dendrogramme calcule la valeur proportionnelle des occurrences des différents concepts et relations mentionnés dans le texte de ce sous-champ sémantique. Par exemple, la ruine et la cause, notés en bleu, sont, respectivement, le concept et la relation sémantiquement les plus fréquents.

Figure 6. Réseau sémantico-logique du champ sémantique 2 « Esthétique » (3D)

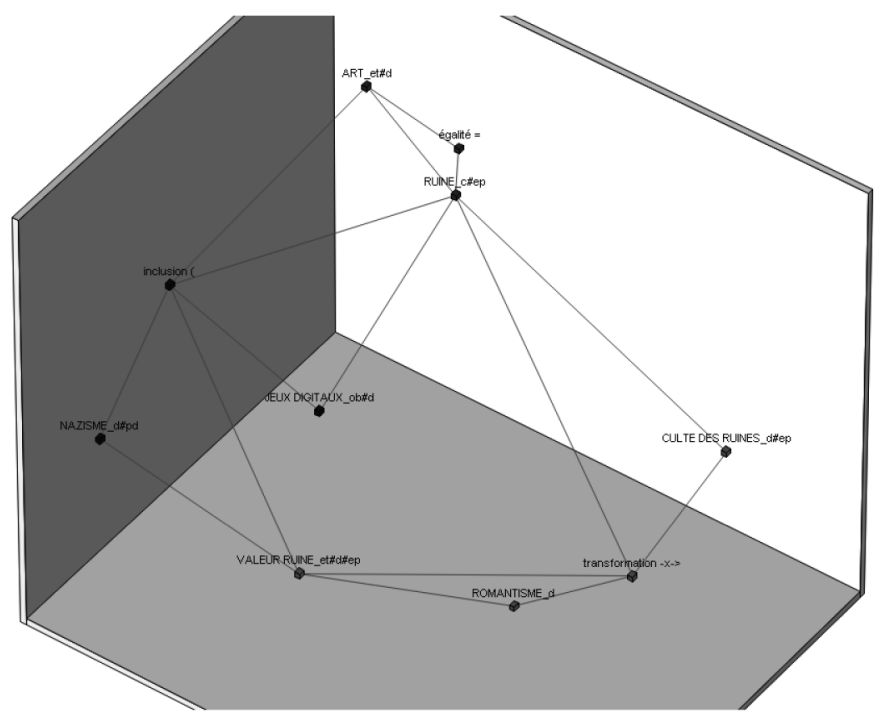

Le même raisonnement peut se produire dans la deuxième section de la page retenue et à l'intérieur du respectif champ sémantique, nommé « Esthétique » (des

10. M. Tsikounas, Imaginaires urbains du Paris romantique à nos jours, Le Manuscrit, Paris, 2011.

11. T. Edensor, Industrial Ruins: Space, Aesthetics and Materiality, Berg Publishers, Oxford, 2005. 
ruines) ${ }^{12}$. Pour la lecture de ce réseau sémantico-logique aux figures 4-7, notons que : 'ob\#d associé au mot 'jeux digitaux', signifie 'object discoursif'; 'd\#pd' lié au terme 'nazisme', veut dire 'discours de pouvoir' ; 'et\#d\#ep', adjoint à l'idée de 'valeur ruine', a la signification de 'structure discursive sur l'espace'. Au sein du réseau sémantico-logique de ce champ, par exemple le sous-champ tissé autour de la relation 'égalité $=$ ' convoye le sens hyperbolique suivant : certains artistes produisent de l'art dont le propos est d'imiter et de se confondre avec des ruines.

Figure 7. Dendrogramme du champ sémantique 2 « Esthétique »
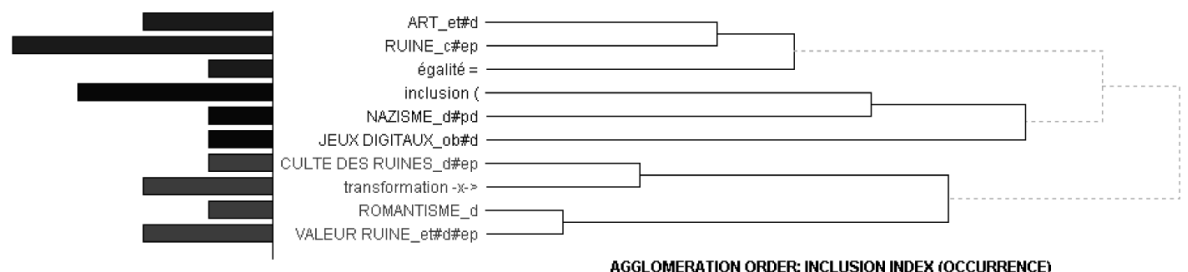

12. Le texte original de cette section est le suivant:

"Aesthetics The ruins of the Parthenon in Athens, Greece. Much of the original marble which formed the roof and frieze now forms a pile of rubble at its base. In the Middle Ages Roman ruins were inconvenient impediments to modern life, quarries for pre-shaped blocks for building projects, or of marble to be burnt for agricultural lime, and subjects for satisfying commentaries on the triumph of Christianity and the general sense of the world's decay, in what was assumed to be its last age, before the Second Coming. With the Renaissance, ruins took on new roles among a cultural elite, as examples for a consciously revived and purified architecture all'antica, and for a new aesthetic appreciation of their innate beauty as objects of venerable decay. [9] The chance discovery of Nero's Domus Aurea at the turn of the sixteenth century, and the early excavations at Herculaneum and Pompeii had marked effects on current architectural styles, in Raphael's Rooms at the Vatican and in neoclassical interiors, respectively. The new sense of historicism that accompanied neoclassicism led some artists and designers to conceive of the modern classicising monuments of their own day as they would one day appear as ruins. Ruin value (German: Ruinenwert) is the concept that a building be designed such that if it eventually collapsed, it would leave behind aesthetically pleasing ruins that would last far longer without any maintenance at all. Joseph Michael Gandy completed for Sir John Soane in 1832 an atmospheric watercolor of the architect's vast Bank of England rotunda as a picturesquely overgrown ruin, that is an icon of Romanticism. [10] Ruinenwert was popularized in the 20th century by Albert Speer while planning for the 1936 Summer Olympics and published as Die Ruinenwerttheorie ("The Theory of Ruin Value"). Ruins remain a popular subject for painting and creative photography [11] and are often romanticized in film and literature, providing scenic backdrops or used as metaphors for other forms of decline or decay. For example, the ruins of Dunstanburgh Castle in England inspired Turner to create several paintings; in 1989 the ruined Dunnottar Castle in Scotland was used for filming of Hamlet. The Civilization series of turn-based strategy computer games features ruins as special tiles which may provide the player with a bonus when explored." 
Une telle esthétique des ruines est manifestée dans plusieurs œuvres qui présentent des connotations spécifiques à l'intérieur de ce champ. Ainsi, les nobles anglais du XIX ${ }^{e}$ siècle construisent des ruines dont le seul objectif est leur contemplation ${ }^{13}$. Les ruines maintiennent également un rapport mélancolique avec les temporalités de la mémoire du cinéma, temps extériorisés souvent sous la forme d'expérience sensible ${ }^{14}$. D'une manière plus générale, cette esthétique possède une généalogie propre ${ }^{15}$. Le culte des ruines se cristallise dans le monument historique, qui témoigne des valeurs conflictuelles conduisant soit à sa restauration soit à son oubli ${ }^{16}$. Mais les ruines peuvent se multiplier par tous les coins du social, afin d'alimenter une vraie industrie du patrimoine ${ }^{17}$. Même l'art a ses ruines, dans le cas du musée, qui est mis en cause par la pratique postmoderne de la provocation culturelle ${ }^{18}$.

Lherméneutique hybride, comme on l'a dit, possède une nature de fusion/ métissage entre les méthodologies qualitative et quantitative. Ce trait de promiscuité est également visible à la figure 5, qui illustre le concept opérationnel hybride nommé intensité sémantique. Il s'exprime empiriquement dans un corpus de sources, d'une part, à travers le pouvoir sémique de chaque concept, visible dans un réseau donné à travers un détail formel. Par exemple, 'Ruine_c\#ep' est le concept hybride le plus fort sémantiquement au sein de la source 'page de Wikipédia' ici analysée. Cette prédominance est exprimée, formellement, par le cercle le plus volumineux dans l'image. D'autre part, du côté des rapports interconceptuels, la relation la plus intense sémantiquement est 'transformation -x->', ce qui est représenté par les lignes les plus larges.

\section{Conclusion}

La ruine fait partie de notre imaginaire social. Toute époque, de la modernité à la postmodernité, peut collectionner ses propres ruines d'estimation. Car la ruine possède un sens constitutif. Par exemple, la modernité s'est érigé, en grande partie, sur la base de sa confrontation avec les ruines de son passé pré-moderne ${ }^{19}$.

Pour le sociologue, les ruines fondent une forme sociale se caractérisant par des traits spécifiques. Ces attributs peuvent s'interpréter à travers des théories et méthodologies séminales, comme la Sociologie sémantico-logique, l'Hybridologie

13. C. Woodwars, In Ruins: A Journey Through History, Art, and Literature, Vintage, London, 2003.

14. A. Habib, Lattrait de la ruine, Yellow Now, Belgique, 2011.

15. R. Mortier, La Poétique des Ruines en France : ses origines, ses variations de la Renaissance à Victor Hugo, Droz, Genève, 1974.

16. A. Riegl, Le culte moderne des monuments, LHarmattan, Paris, 2003.

17. J. Jackson, The Necessity for Ruins: And Other Topics, University of Massachusetts Press, Boston 1980.

18. D. Crimp, On The Museum's Ruins, The MIT Press, Massachusetts, 1995.

19. J. Hell \& A. Schonle (Editors), Ruins of Modernity, Duke University Press Books, 2010. 
sociale ou l'Herméneutique hybride. Lobjectif étant de traiter la ruine, comme disait Derrida, non pas comme le résultat d'un processus, monumental ou autre, mais en tant qu'origine, fournissant le simulacre ruineux de l' « autoportrait ». Cette " mémoire ouverte ${ }^{20}$ peut informer d'un mode pluriel l'œil du sociologue qui, comme tous les autres acteurs sociaux, est aujourd'hui un être inévitablement hybride.

\section{Bibliographie}

Andrade P., Sociologia Semântico-Lógica da Web 2.0/3.0 na sociedade da investigação: significados e discursos quotidianos em blogues, wikis, mundos/museus virtuais e redes sociais semântico-lógicas, Libsonne, Caleidoscópio, 2011.

Andrade P., "O alfabeto das relações universais ", Revista de Comunicação e Linguagens, 38, 2007, pp. 143-155.

Andrade P., «Some synthetic ideas to work with qualitative analysis », Atalaia/Intermundos, 10/11, 2002, pp. 153-165.

Camacho S., Chernobil: 25 Anos Despues, Debate Editorial, Madrid, 2011.

Crimp D., On The Museum's Ruins, The MIT Press, Cambridge, 1995.

Derrida J., Mémoires d'aveugle : l'autoportrait et autres ruines, Réunion des musées nationaux, Paris, 1991.

Edensor T., Industrial Ruins: Space, Aesthetics and Materiality, Berg Publishers, Oxford, 2005.

Habib A., Lattrait de la ruine, Yellow Now, Belgique, 2011.

Hell J. \& Schonle A. (Eds.), Ruins of Modernity, Duke University Press Books, Durham \& Londres, 2010.

Jackson J., The Necessity for Ruins: And Other Topics, University of Massachusetts Press, Boston, 1980.

Lacroix S., Ce qui nous disent les ruines : fonctions critiques des ruines, LHarmattan, Paris, 2007.

La Rocca F., « Habitar a Web: paisagens e nuvens da cultura digital », Revista de Comunicação e Linguagens: Genealogias da Web 2.0, 42, 2011, pp. 53-58.

Maffesoli M., La passion de l'ordinaire : Miettes sociologiques, CNRS Éditions, Paris, 2011.

Mortier R., La Poétique des Ruines en France : ses origines, ses variations de la Renaissance à Victor Hugo, Droz, Genève, 1974.

Riegl A., Le culte moderne des monuments, LHarmattan, Paris, 2003.

Tsikounas M., Imaginaires urbains du Paris romantique à nos jours, Le Manuscrit, Paris, 2011.

Woodwars C., In Ruins: A Journey Through History, Art, and Literature, Vintage, London, 2003.

20. J. Derrida, Mémoires d'aveugle : l'autoportrait et autres ruines, Réunion des musées nationaux, Paris, 1991, p. 72. 